Int. J. Dev. Biol. 49: 527-537 (2005)

doi: $10.1387 /$ ijdb.041967ja

\title{
Flower symmetry and shape in Antirrhinum
}

\author{
JORGE ALMEIDA* ${ }^{*}, 1,2$ and LISETE GALEGO ${ }^{2}$ \\ ${ }^{1}$ Instituto Superior de Agronomia (ISA), Lisboa, Portugal and ${ }^{2}$ Instituto de Tecnologia Química e Biológica (ITQB), Oeiras, Portugal.
}

\begin{abstract}
According to their symmetry, flowers are classified as radially symmetrical or bilaterally symmetrical. Bilateral symmetry, which is thought to have evolved from radial symmetry, results from establishment of asymmetry relative to a dorsoventral axis of flowers. Here we consider developmental genetic mechanisms underlying the generation of this asymmetry and how they relate to controls of petal shape and growth in Antirrhinum. Two genes, CYC and DICH, are expressed in dorsal domains of the Antirrhinum flower and determine its overall dorsoventral asymmetry and the asymmetries and shapes of individual floral organs, by influencing regional growth. Another gene, DIV, influences regional asymmetries and shapes in ventral regions of the flower through a quantitative effect on growth. However, DIV is not involved in determining the overall dorsoventral asymmetry of the flower and its effects on regional asymmetries depend on interactions with $C Y C / D I C H$. These interactions illustrate how gene activity, symmetry, shape and growth may be related.
\end{abstract}

KEY WORDS: plant development, antirrhinum, shape, growth, dorsoventral asymmetry

\section{Introduction}

The shapes of organisms and of their parts are often described in terms of symmetries. Clearly, however, symmetry is not equivalent to shape, since instances of diverse organisms or parts with the same symmetries and different shapes can be readily found. Even within species, a level at which symmetry is largely invariant, details of shape may mark distinctions among individuals (for example, there are many different shapes for the bilaterally symmetrical human head). This implies that mechanisms generating finely grained differences in shape will often leave symmetries unaltered, suggesting that symmetry and shape are uncoupled. An alternative view is that symmetry and shape are related, when considered as properties that change during development and evolution. This is supported by studies on developmental genetic mechanisms underlying the generation of asymmetries during flower and leaf development in Antirrhinum majus (reviewed in Coen, 1996; Hudson, 2000; Schwarz-Sommer et al., 2003). The two views are, however, not mutually exclusive and may be conveniently introduced further by considering symmetries in geometrical figures.

According to an informal Mathematical definition, a symmetry of a geometrical figure or object is a motion which, when applied to the object, results in a figure that looks exactly the same as the original (Scheinerman, 2000). In this definition, it is implied that the motion is one that goes undetected by an observer who has access only to the initial and final states of the figure. For example, a regular pentagon has 5 rotational symmetries because it can undergo 5 rotations about its centre, each of which leaves it exactly as it was (Fig. 1A). In addition, it has 5 flip (reflection or mirror) symmetries because, again without change in its appearance, it can be flipped relative to any of the 5 axes containing a vertice and the mid-point of the side opposite to that same vertice. In contrast, the irregular pentagon shown in Fig. $1 \mathrm{C}$ has only one symmetry, equivalent to undergoing no motion (a trivial symmetry). Another irregular pentagon (in Fig. 1B) has bilateral symmetry, i.e., one trivial rotational symmetry and one mirror symmetry.

Objects can be classified in two types according to their numbers of rotational and mirror symmetries. Objects with only rotational symmetries are classified as Cyclic or of C-type followed by a number indicating how many symmetries they have. Objects with both rotational and mirror symmetries have Dyad or D-type symmetry, followed by the number of their mirror symmetries (since the numbers of rotational and mirror symmetries are identical in such objects). Thus, for example, the pentagons in Fig. $1 \mathrm{~A}, \mathrm{~B}$ and $\mathrm{C}$ are of type D5, D1 and C1, respectively. Such classification is useful because it allows objects to"be compared. For example, objects $1 \mathrm{G}$ and $1 \mathrm{H}$ are different but have a common D5 symmetry. They differ in the shapes of the so-called basic or cell units of the sets (groups) of their symmetries, the red sectors which can cover the entire figures without overlaps when subjected to the flips and rotations described above (Farmer, 1996). Conversely, the same cell unit (e.g., that of Fig $1 \mathrm{H}$ ) could be arranged to give different symmetry types (D5 in Fig. $1 \mathrm{H}$ and C5 in Fig.1I). Comparisons of rigid objects might therefore suggest that shape is a property of cell units while symmetry describes

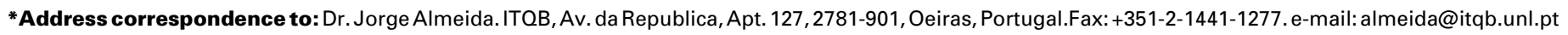




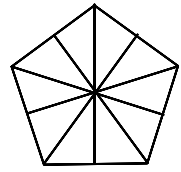

A

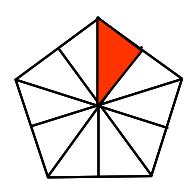

D

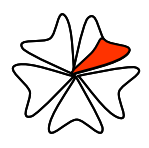

G

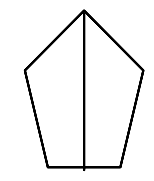

B

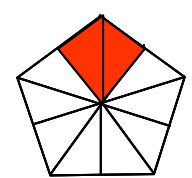

E
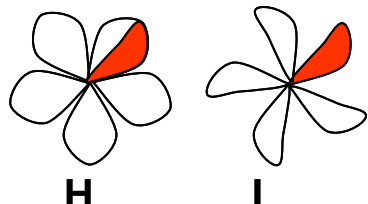

Fig. 1. Symmetries of geometrical figures. The red sectors represent the basic units of the groups of all symmetries in D, G and H, or of only the 5 rotation or reflexion symmetries in $E$ and $F$. Note that the sectors in $E$ or $F$ can cover the regular pentagons when subjected to either rotations or flips. The basic unit of all symmetries is itself asymmetrical (D, G, H and I).

arrangements (or, more accurately, permutations) of cell units, hence that symmetries and shapes are unrelated.

As mentioned above, this may in many instances apply to biological structures considered in a static manner. However, unlike rigid objects, living structures do undergo changes in symmetry and shape during development and evolution. Many of these changes may involve equal modification in the shapes of all basic units of a structure leaving symmetries unaltered while others may alter numbers of symmetries without necessarily modifying the shapes of basic units. Other changes in symmetry, however, may be coupled to differential modification of the shapes of basic units. Two ways for how such modification might arise can be illustrated by considering transformations of a growing regular pentagon along one of its axes of symmetry. One way involves modifying the shapes of all basic units in a coordinated manner, as when the"entire regular pentagon is stretched along a vertical axis (Fig. 2A). Another way is through modification of only a subset of the basic units (Fig. 2B). In either case, only the axis along which the transformation operated remains an axis of mirror symmetry. This axis is therefore both of bilateral symmetry and of vertical asymmetry.

\section{Flower symmetry and asymmetry}

A range of floral symmetries can be identified according to the classification above for geometrical figures (considering planar projections of flowers). However, flowers have traditionally been classified in two basic types according to their symmetry: Radially symmetrical flowers, also known as regular or actinomorphic, which have two or more non-trivial symmetries and bilaterally symmetrical flowers (irregular or zygomorphic), which have a single mirror or reflexion symmetry. There are also rare cases of flowers without non-trivial symmetries (C1), which fall in neither of the two basic classes (not considered here, see Endress, 2001). The regular class includes a range of D-types (e.g., D3 in many monocots, D4 in Arabidopsis) and also some C-types (e.g., Oleander flowers are of type C5, as in Fig. 1I). The irregular class can only include the D1 type. However, many variants can be recognized within this type when regional symmetries are taken into account. For example, amongst bilaterally symmetrical pentameric corollas ( 5 petals), there can be those with two or three different types of petals. Further variants can be identified when internal symmetries of individual petals are considered.

Bilaterally symmetrical flowers are also described as dorsoventrally asymmetrical (Carpenter and Coen; 1990, Coen, 1991). The different designations are equivalent but reflect distinct ways of looking at flowers. Looking at either of the flowers in Fig. 3B from left to right leads to recognition of mirror image halves, hence symmetry. Another way of looking at the same flowers is from top to bottom. In this case, vertical asymmetry is readily perceived in the bilaterally symmetrical flower. This connection between flower symmetry and asymmetry may be considered further by looking at flowers as parts of developing plants.

All aerial parts seen in mature plants are generated from the apical meristem. Early in plant development this meristem is vegetative, generating leaves before undergoing a transition to a reproductive (floral) phase. In some species the apical vegetative meristem is directly converted into a floral meristem, giving rise to terminal flowers that are usually radially symmetrical. In other species, however, the vegetative meristem is converted into an inflorescence meristem on the periphery of which floral meristems then can develop (Coen et al., 1995; Bradley et al., 1996). These lateral floral meristems give rise to bilaterally symmetrical flowers in some species while in others they give radially symmetrical flowers. What is common to both symmetry types arising from lateral meristems and different from terminal flowers, is that floral organs will in general be aligned with an axis directed from the centre to the periphery of the apex (or up-down, see Fig. 3). This
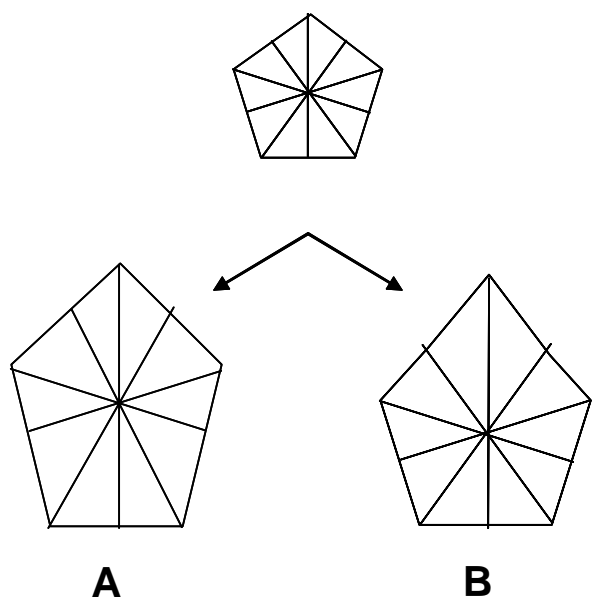

Fig. 2. Converting a D5 figure into D1 figures. In (A), the pentagon grew preferentially along the vertical axis. Out of the 5 triangles (corresponding to basic units of mirror symmetry) composing the pentagon, all but the lower one have become internally asymmetrical and all have changed shape. In (B), only a top unit (of mirror or rotational symmetry) has changed shape. 
A

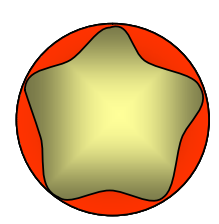

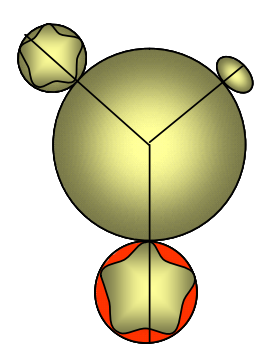

B
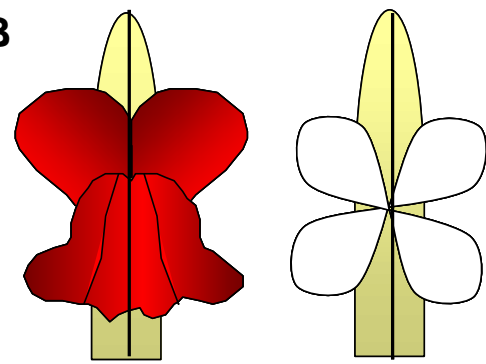

Fig. 3. Plant architecture and dorsoventrality of flowers. (A) Large yellow circle represents the apical meristem viewed from above. Floral organs are in red. Terminal flower on the left and inflorescence meristem with lateral flowers on the right. (B) Side view of inflorescence meristems with a dorsoventrally asymmetrical flower (left) or a radially symmetrical flower (right), showing alignment of petals with dorsoventral axis. alternate with the petals, are also of three types: Two long ventral stamens, two short lateral ones and a staminoid (remnant of a developmentally arrested stamen) at the most dorsal position. This pattern is generated progressively during flower development, a sequence of events that can be divided in several stages according to time of emergence, size and morphological details of the floral meristem and floral organs (Carpenter and Coen, 1995; Vincent and Coen, 1995; 2004).

During stages 1-2, floral meristems showing no obvious dorsoventral asymmetry arise and grow in the periphery of the apical inflorescence meristem (Fig. 5). While at these stages the floral meristem appears to have two planes of mirror axis, which coincides with a plane of floral symmetry in both cases, is termed a dorsoventral axis, dorsal (or adaxial) being towards the apical inflorescence meristem. Unlike in radially symmetrical lateral flowers, in bilaterally symmetrical flowers this axis is an axis of asymmetry.

The term dorsoventrality has also been applied to lateral organs, such as leaves or petals, to describe differences following an axis at a right angle to their surfaces. In this case, positions are defined relative to the meristem that generated the organ, the dorsal side of a petal, for example, being the one facing the centre of the floral meristem (Waites etal., 1998; Sessions and Yanofsky; 1999; Waites and Hudson, 2001). This is unlike the meaning of dorsoventrality here, in which positions of individual organs or within organs are defined relative to the dorsoventral axis of the flower (hence, relative to the inflorescence meristem that generated the floral meristem).

Bilateral symmetry is thought to have evolved several times independently from radial symmetry, possibly in relation to pollination by animals (Coen and Nugent, 1994; Neal. et al., 1998; Cronk, 2001; Rudall and Bateman; 2004). Presumably, transitions from radial to bilateral symmetry occurred in species with lateral flowers, that is, with a dorsoventral floral axis. Repeated evolution may therefore reflect that there are various ways by which that axis may be rendered an axis of asymmetry, an analogy being the situation illustrated in Fig. 2 where modifications along a given axis converge in bilateral symmetry. This might result in the diversity of dorsoventral patterns exhibited by bilaterally symmetrical flowers. Alternatively, this diversity reflects elaborations on a common mechanism that could have arisen repeatedly. So far, however, genetic and developmental mechanisms underlying dorsoventral asymmetry of flowers have been investigated in detail only in Antirrhinum majus, as considered next.

\section{Dorsoventral asymmetry in Antirrhinum flowers}

Dorsoventral asymmetry is particularly clear in whorls 2 (petals) and 3 (stamens) of the Antirrhuinum flower. The corolla has 5 petals of 3 identities according to their positions relative to the dorsoventral axis of the flower: One ventral, a pair of lateral and a pair of dorsal. The ventral petal is symmetrical about the plane of bilateral symmetry of the flower whereas each of the dorsal and lateral petals is internally asymmetrical (Fig. 4). Stamens, which symmetry, at stage 3 it adopts a nearly regular pentagonal shape with 5 planes of symmetry. These planes will in subsequent stages coincide with divisions of organs in two halves or with boundaries between organs of the same whorl. By stage 4, sepal primordia are visible in the periphery of the floral meristem (whorl 1), located at precise positions: One dorsally, two laterally and two ventrally. Growth of the dorsal primordium is somewhat retarded relative to the remaining primordia, an early manifestation of dorsoventral asymmetry that becomes attenuated in mature flowers.

Stage 5 is characterized by the emergence of petals and stamens in whorls 2 and 3 respectively. As petal primordia alternate with the sepals that were formed on the first whorl, there is one ventral, two lateral and two dorsal petals. Similarly, organs in whorl 3 alternate with those in whorl 2, a single stamen primordium therefore being placed dorsally. By stage 6 , when two carpel primordia arise in the centre of the meristem, dorsoventrality is already clear, with the dorsal stamen primordium appearing retarded in growth relative to other stamens and the dorsal petals differing in shape from the remaining 3 petals. Primordia of the ventral and lateral petals remain however very similar and all individual organs have a bilaterally symmetrical appearance (Fig. 5). Through stages 7-8, the ventral petal becomes different from the lateral ones and the internal asymmetries of lateral and dorsal petals become manifest. These internal asymmetries then increase as the flower continues to grow in subsequent stages. It appears therefore that asymmetry is generated in a stepwise

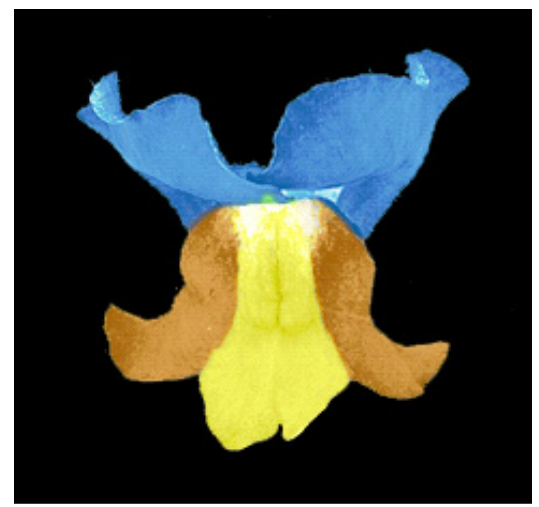

Fig. 4. Antirrhinum flower corolla with petals coloured according to identities. Ventral in yellow, lateral in brown and dorsal in blue. 
manner, with distinctions arising first between organs (e.g., dorsal vs. lateral-ventral and then ventral vs. lateral in the corolla) and later within organs.

\section{Genes controlling dorsoventral asymmetry}

Most of what is known on genetic mechanisms underlying the dorsoventral pattern described above comes from the analysis of mutants with altered symmetry. Many of these were spontaneous mutants occurring in wild or cultivated plants that had since long caught the attention of Naturalists. Already in the eighteenth century, Linnaeus had become intrigued with the finding of a naturally occurring variant of Linaria with radially symmetrical flowers (Linnaeus, 1749; Gustafsson, 1979; Cubas etal., 1999b). Linaria normally has bilaterally symmetrical flowers, which except for the presence of a long spur at the base of the corolla are very similar to those of Antirrhinum, one of its closest relatives. Linnaeus coined the term peloria, after the Greek word for monstrosity, to describe the radially symmetrical Linaria, which he took as an indication of the mutability of species.

Later in the nineteenth century it was Darwin's turn to attend to the phenomenon of peloria in various species, including a variant of Antirrhinum known as "Wonder". Darwin thought of peloria as revealing an ancestral condition of species with irregular flowers and dismissed a view then held, according to which peloria represented an arrest in the development of floral parts (Darwin, 1868). To come to this view, Darwin

$1-2$

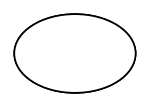

3

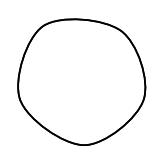

5-6

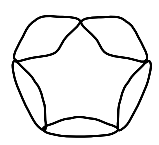

7-8

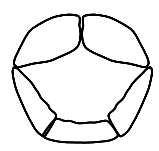

$>9$

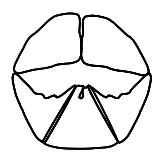

Fig. 5. Schematic representation of stages of corolla development in Antirrhinum (stages indicated on the left, see text), showing the generation of differences between petals and of asymmetries in individual petals. Growth is ignored in this representation. misplaced. For example, in the deficiens mutant of Antirrhinum sepals appear at the place of petals and carpels at the place of stamens and in ovulata mutants carpels replace sepals and stamens replace petals (Sommer et al., 1990; Coen and Meyerowitz, 1990; Schwarz-Sommer et al., 1990; Bradley et al., 1993). The meristic series is in both of these cases defined following an axis of asymmetry from the centre to the periphery of the flower (leading to a whorled sequence of sepals, petals, stamens and carpels in normal flowers). By contrast, in radially symmetrical mutants the series may be defined relative to the dorsoventral axis of the flower, with some organs adopting the character and shape of other organs of the same rather than of a different type. Although this implies that such homeotic effects will often be subtle, mutant peloric phenotypes could be readily interpreted. As already hinted at by Darwin for the case of Galeobdolum, in peloric flowers of Antirrhinum all members of a whorl resemble the wild type ventral (lower) member of the same whorl (e.g., all members of the corolla have ventral petal identity). An advantage of describing peloric flowers in terms of the phenomenon of homeosis was that, as shown below, it would provide a means for seemingly disparate mutants to be related, leading to the identification of four genes, $C Y C, D I C H, R A D$ and $D I V$ which control the dorsoventral pattern of Antirrhinum flowers.

\section{CYCLOIDEA and DICHOTOMA}

Null cyc mutants have a so-called semipeloric phenotype in which ventral and lateral petals all have ventral identity and the dorsal petals have latero-dorsal identity (Luo et al., 1996, Fig. 6). A similar corolla phenotype, not considered here, is caused by mutation in the RADIALIS gene (Carpenter and Coen, 1989). In stamens, $c y c$ mutants also show changes that are in register with those in the corolla. In this case, a stamen with lateral identity develops at the most dorsal position and the remaining stamens show ventral identity. Mutation at $C Y C$ therefore has a ventralizing effect, indicating that $C Y C$ is required for determining dorsal and lateral identities. Consistent with this view, $C Y C$ mRNA occurs specifically in a dorsal domain of the wild type floral meristem and later on in dorsal organs (staminoid and dorsal petals). However, cyc transcripts are undetectable in lateral petals, suggesting a non-cell autonomous effect in lateral regions (Luo et al., 1996).

Another effect observed in most flowers from cyc mutant plants is an increase in organ numbers from 5 to 6 in whorls 1,2 and 3 , consistent with a role of $C Y C$ in inhibiting primordium initiation or growth. This becomes apparent as soon as sepal primordia start to emerge (stage 4). An additional early effect, which is detected in some flowers from cyc mutant plants with increased organ numbers, is a misalignment of the plane of bilateral symmetry with the axis of dorsoventral asymmetry, as defined relative to the apical inflorescence meristem (Fig. 6). This has suggested that $C Y C$ is required for bringing organ positions into register with the axis of asymmetry, an effect that might be coupled to growth inhibition (Luo et al., 1996).

In fully peloric phenotypes all petals and stamens adopt ventral identity. Thus, in considering a transformation from semipeloric to peloric only the dorsal petals and the single most dorsal stamen become altered. The two phenotypes are therefore obviously related and have since long been known to require mutation at the $c y c$ locus. However, only relatively recently has the basis for the difference between peloric and semipeloric been uncovered 


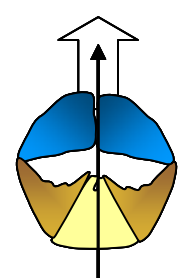

A

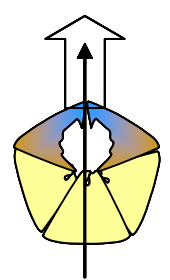

B

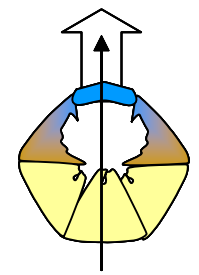

C

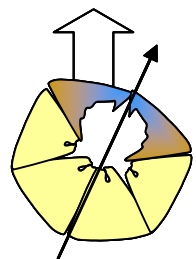

D

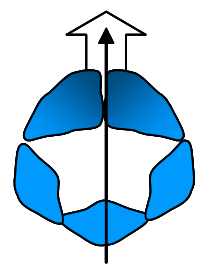

E
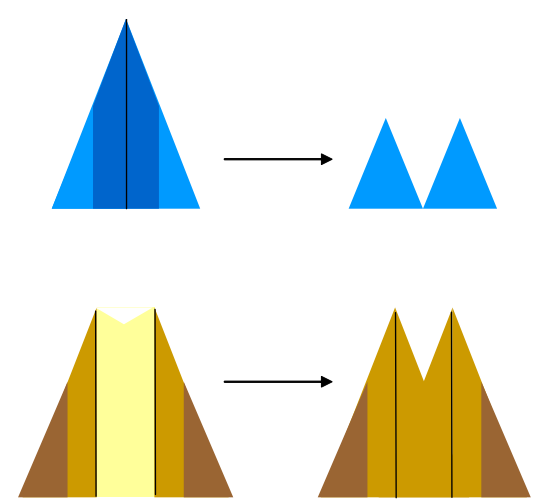

Fig. 6 (Left). Schematic representation of phenotypes of flowers from wild type (A) and from cyc mutant plants (B-E). Colours represent identities as in Fig. 5. Petals with latero-dorsal identity are represented by a gradient from brown to blue. In mutants, 6 petals (C,D) occur more often than 5 (B). In some cases (C), flowers have 3 petals with ventral identity. In other cases they have 4 petals with ventral identity (D) and with the plane of bilateral symmetry (thin arrow) misaligned with the dorsoventral axis (white arrow). Unlike (B,C and D, which involve loss of CYC function), (E) represents backpetals, a cyc gain-of-function mutant.

Fig. 7 (Right). Schematic representation of effects of dich (top) and div (bottom). At the top, dorsal petals are separated by a vertical black line. Each petal is divided into a dorsal half (dark blue) and a more lateral half (paler blue). In each of the dorsal petals of dich mutants, the dark blue region is replaced by a mirror-image duplication of the pale blue region. At the bottom the ventral petal is in yellow. The lateral petals are each divided into a ventral part (pale brown) and a lateral part (dark brown). In weak div mutants each half of the ventral petal is replaced by a mirror-image duplication of the adjacent (pale brown) region of the lateral petal.

through a combination of genetic and molecular analyses. These have shown that the fully peloric phenotype is that of double mutants for $C Y C$ and an additional gene, DICH (Luo et al., 1996; 1999; Coen, 1996; Almeida et al., 1997).

Mutation at dich affects specifically the dorsal parts of the flower without any obvious effect on dorsal or lateral parts. In one description, the asymmetry of the dorsal petals becomes reduced and the staminoid grows longer relative to wild type. Reduction in asymmetry becomes obvious when, in particular genetic contexts, mutations in dich cause otherwise asymmetrical petals to become symmetrical. One case is, as mentioned above, that of considering the transformation brought about by inactivation of dich in a cyc mutant background which causes asymmetrical dorsal petals (in single $c y c$ ) to become symmetrical (in double cyc;dich ). Another case is deduced from the phenotype of a dominant $c y c$ mutant known as backpetals, in which $C Y C$ is expressed ectopically in lateral and ventral petals (Luo et al., 1999, Fig. 6). These petals do not express $D / C H$ and have dorsal identity, but are symmetrical. Their symmetry and shape can be readily simulated by replacing, within a normal wild type dorsal petal, the dorsal half by a mirror-image duplication of the lateral half (Fig. 7). Finally, a third case, comes from the observation of symmetrical dorsal petals, which can be generated by the same mirror-image duplication as in backpetals, caused by mutation of dich in some ill-defined genetic backgrounds (our unpublished observation).

Taken together, these observations indicate that $\mathrm{D} / \mathrm{CH}$ is active in the dorsal half of the dorsal petals. Consistent with this view, $D / C H$ is expressed in a dorsal domain of the floral meristem similarly to $C Y C$ and later on in dorsal organs. However, whereas at later stages of flower development $C Y C$ is expressed in both halves of the dorsal petals, $\mathrm{D} / \mathrm{CH}$ expression becomes restricted to their dorsal halves (Luo et al., 1999). This narrowing of the domain of $\mathrm{D} / \mathrm{CH}$ expression correlates with the region affected in mutants and with the generation of internal asymmetry in the dorsal petals which becomes manifest relatively late (by stage 8).

In line with a redundant role in generating dorsoventral asymmetry of the flower, $\mathrm{CYC}$ and $\mathrm{DICH}$ encode related proteins belonging to the TCP family of transcription factors (Luo et al., 1996; 1999; Cubas et al., 1999a). This redundancy relates to two ways of generating dorsoventral asymmetry, as can be seen by considering the consequences of adding either gene function to radially symmetrical mutants (reversing the arrows and signs in Fig. 8). Addition of $C Y C$ changes the shapes and symmetries of all but the ventral petal while addition of $D / C H$ alters only the dorsal petals. As argued below, in wild type each half of the ventral petal retains some symmetry relative to the adjacent region of the lateral petal (this becomes more obvious in a div mutant background). This implies that adding $\mathrm{DICH}$ to the radially symmetrical condition changes mostly the dorsal parts of the dorsal petals, rather than the entire petals, similarly to what has been illustrated in Fig. 2B as a way of generating bilateral from radial symmetry through change in the shape of a basic unit of the set of mirror symmetries.

\section{DIVARICATA}

The observation that $c y c$ mutants are ventralized suggested that $C Y C$ might have a role in inhibiting ventral determinants. This led to a search for mutants in which ventral parts of the corolla would be specifically affected. One such mutant, div, since long part of Antirrhinum collections, turned out to have a phenotype comparable to that of $c y c$, as some regions of the corolla ap- 
peared to adopt identities of other regions (Schick and Stubbe, 1934, Almeida et al., 1997). In contrast to $c y c$, however, in div mutants the ventral petal adopts lateral identity. Thus, the domain and polarity of the div effect are opposite to those of cyc. This is particularly clear in weak div mutant phenotypes which, similarly to what was described above for dich, can be simulated by replacing each half of the ventral petal by a mirrorimage duplication of the adjacent region in the lateral petal (Fig. 7). In null div mutants, such lateralizing effect is more pronounced, with a domain spanning the ventral petal and adjacent parts of the lateral petals taking on a more lateral identity.

DIV has no obvious effect on parts of the flower other than ventral and lateral petals or on the timing of petal initiation. Therefore, its influence on morphology becomes apparent only after stage 5 of flower development when petals are initiated. Through stages 6-8, the ventral petal remains very similar to the lateral ones in null div mutants, unlike in wild type where differences become manifest at these stages. From stage 9 onwards, the lateral petals of flowers from div mutant plants become asymmetrical. However, this asymmetry is much less pronounced than in flowers from wild type plants. These effects depend quantitatively on levels of DIV activity. The wild type allele is haploinsufficient and therefore heterozygotes for $D / V$ and null mutant div alleles, presumably showing a $50 \%$ reduction in activity, confer the weak phenotype described above. The same phenotype can be conferred by weak mutant alleles in the homozygous condition. Thus, by combining the three types of alleles, a series with progressively increased lateralization can be produced (Almeida et al. 1997, Galego and Almeida, 2002).

The DIV gene encodes a protein belonging to the MYB family of transcription factors (Galego and Almeida, 2002). DIV is transcribed in inflorescence and floral meristems and at early stages of development of all floral organs irrespective of whorl and dorsoventral position. However, from stage 8 onwards, DIV transcription appears to be strongly induced in the inner layer of epidermal cells, in a domain that spans the ventral petal and adjacent parts of the lateral petals, that is, in register with the region affected in mutants. DIV activity precedes the establishment of this pattern. In addition, the pattern fails to be established in null, transcribed, div mutants. This has suggested that early $D / V$ activity may itself be needed for setting up the late pattern of $D I V$ transcription.

\section{Dorsoventral interactions}

Interactions between DIV and the dorsal genes have been analysed through mutant combinations. As seen above, in cyc;dich double mutants all petals have ventral identity, which requires $D I V$ activity. This suggested that the domain of DIV activity, normally restricted to ventral and lateral petals, spreads all around the flower in cyc;dich double mutants. One prediction therefore was that inactivation of $D I V$ in a radially symmetrical context would alter the shapes of all petals. This is indeed what is observed in triple cyc;dich; div mutants which have radially symmetrical flowers in which all petals have lateral identity (Almeida et al., 1997, Fig. 8). Formally, therefore, the dorsal genes inhibit DIV. Consistently with this, the late pattern of DIV transcription spreads all around the flower in cyc;dich double mutants (Galego and Almeida, 2002). This interaction must however be indirect. First, because $C Y C$ and $D / C H$ encode transcription factors and $D I V$ is transcribed in all petals through stages of flower development when all 3 genes are active. Second, $C Y C$ has an effect on the symmetry of lateral petals, which is independent of $D I V$ (see Fig. 8).

The phenotypes of mutant combinations illustrate how symmetry and shape can be related to gene activities. By adding DIV to a triple mutant condition, neither the overall symmetry of the flower nor the symmetries of individual organs are altered. What becomes modified is the shape of the basic unit of the set of all symmetries, that is, each half petal. Thus, unlike $C Y C / D / C H, D I V$ would qualify as a shape gene, rather than a symmetry gene. However, adding DIV to an otherwise wild type background results in increased asymmetry within lateral petals and between lateral and ventral petals, reflecting interactions between DIV and the symmetry gene $C Y C$.

\section{Continuous description of flower shape and symmetry}

Terms such as nearly actinomorphic and strongly or weakly zygomorphic are often used in Botanical descriptions. Similarly, some regions of the flower, such as individual petals, are referred to as strongly or weakly asymmetrical or as increasing or decreasing in asymmetry during development or as a result of gene muta-

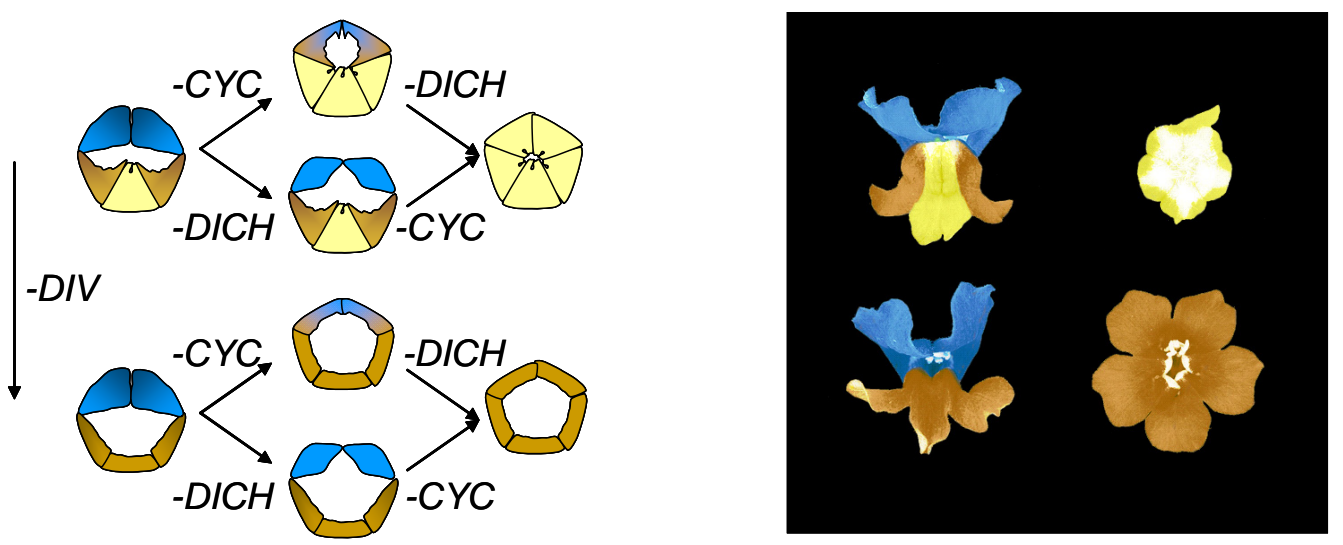

Fig. 8. Summary of phenotypes conferred by combinations of cyc, dich and div. Schematic (left) and actual (right) corollas coloured as in Fig. 7. tion (e.g., our own descriptions). Implicit in these terms is an intuitive notion of quantitative or continuous variation in symmetry and that symmetry can be broken into regional components. However, as described before, most Mathematical treatments of symmetry are discrete, with objects being classified according to their types (e.g., mirror, rotational) and numbers of symmetries. Thus, mathematically, an object being more symmetrical than another simply means that it has more symmetries (a regular hexagon is more 

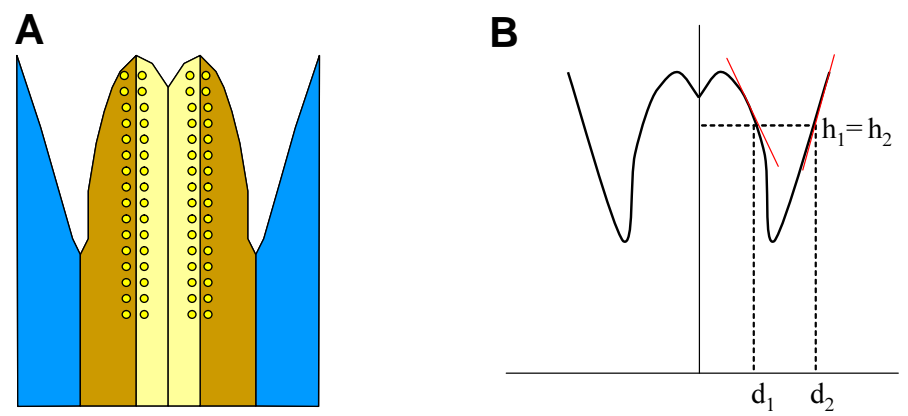

Fig. 9. Continuous description of flower shape. (A) Representation of the internal surface of the Antirrhinum flower tube. The tube is dissected from the lobes, cut dorsally along the proximo-distal axis and then flattened. Therefore, dorsal is to the sides and ventral is in the middle. Proximal is at the bottom. Petals coloured as in Fig. 7. The yellow circles represent cell types placed at symmetrical positions relative to the ventro-lateral boundaries. (B) Tube height (h) as a function of distance (d) from the dorsoventral axis. The red lines indicate slopes ( $h$ ', see text).

symmetrical than a regular pentagon). It is therefore unclear how flower symmetries might be described quantitatively.

One approach is suggested by detailed analysis of the Antirrhinum flower corolla. The five petals are fused at the base (proximally) forming a tube that ends distally in a sharp border with the lobes. The length, or height, of the tube varies in a continuous manner according to position relative to the dorsoventral axis (Fig. 9A). From a peak at the most dorsal position, tube height decreases continuously to a deep trough at the boundary between dorsal and lateral petals, then increases to a new peak at the boundary between lateral and ventral petals and finally comes down to a shallow trough at the plane of symmetry of the ventral petal.

The distal contour of the tube is therefore a function, $h=f(d)$, which describes how tube height $(h)$ varies according to position (d) relative to the dorsoventral axis (Fig. 9B). As the function is continuous, the values that it adopts at different positions might be used to describe shape in infinitesimal detail. Clearly, however, these values are insufficient for describing shape. For example, to two different positions such as $\mathrm{d} 1$ and $\mathrm{d} 2$ in Fig. 9B can correspond the same value of $h(h 1=h 2)$, even though the lateral region containing $\mathrm{d} 1$ is manifestly different from the dorsal region containing $\mathrm{d} 2$. This difference can however be expressed by taking into account slopes (h', first derivatives of $h$ ) which measure the way in which tube height is changing regionally (or infinitesimally). Although the function adopts the same value (h1=h2), it has different slopes (h'1 and h'2) for positions d1 and $\mathrm{d} 2$ respectively. Each position (di) has therefore an identity that can be described quantitavely by a pair of values (hi, h'i).

A continuous view leads to a more detailed description of regional symmetries. For example, in wild type the ventral petal is apparently distinct from the lateral petal. However, as measured by pairs of values ( $\left.h, h^{\prime}\right)$, the magnitude of differences between regions located to either side and at identical distances from the ventro-lateral boundary decreases continuously with decreasing distance from the boundary (this should apply to any maximum or minimum of the function, that is, to all boundaries). This implies that the ventro-lateral boundary coincides with an axis of infinitesimal symmetry. Theoretically, this symmetry is broken at an infinitesimal distance to either side of the boundary. In practice, however, some discrete regional symmetry is retained, being manifest, for example, in the presence of unique cell types located at symmetrical positions relative to the boundary (Fig. 9A, Almeida et al., 1997).

This view may account for how, by acting quantitatively, a shape gene such as $D / V$ influences regional symmetry between lateral and ventral petals. As $D I V$ activity is progressively reduced in mutants, a pre-existing symmetry is increased rather than generated from complete asymmetry. This requires only that $D / V$ controls tube growth mostly in height and to different extents at distinct positions relative to the dorsoventral axis. For example, reduction by about $50 \%$ in $D / V$ activity causes an increase in the slope of each half of the ventral petal tube extending the range in which its values are symmetrical (similar absolute values with opposite sign) to those in the adjacent region of the lateral petal (Fig. 10). One way in which this effect can be mimicked is by anchoring tube height both at the ventro-lateral and latero-dorsal boundaries and bringing down height at the plane of bilateral symmetry. Similarly, the effect of further reduction in DIV activity (towards the null condition) may be mimicked by generalized reduction in height in ventral and lateral petals while maintaining tube height anchored at the boundary between lateral and dorsal petals. An outcome is reduction of the asymmetry of lateral petals, which may be seen in the continuous approach as a reduction in the average slope of the height function.

Anchoring at the latero-dorsal boundary corresponds to inhibiting tube growth at that position through the activity of the dorsal gene $C Y C$. This results in a trough, which in the absence of $C Y C$ activity becomes a peak with ventral or lateral identity depending on DIV (Fig. 10). In double cyc;dich mutants, the height function becomes periodic with maxima and minima at petal boundaries and medial petal planes respectively. Mechanisms underlying the generation of this function are unknown. One possibility is that its basic shape (in triple cyc;dich;div mutants) depends mostly on the extreme values that it adopts at boundaries and medial planes as a result of interactions of growth determinants. Between extremes, the function would take intermediate values through some non-autonomous mechanism. This would readily explain the shape and asymmetry of the lateral petal tube, if DIV stimulates growth at the ventro-lateral boundary and $C Y C$ inhibits growth at the latero-dorsal boundary, through interactions with

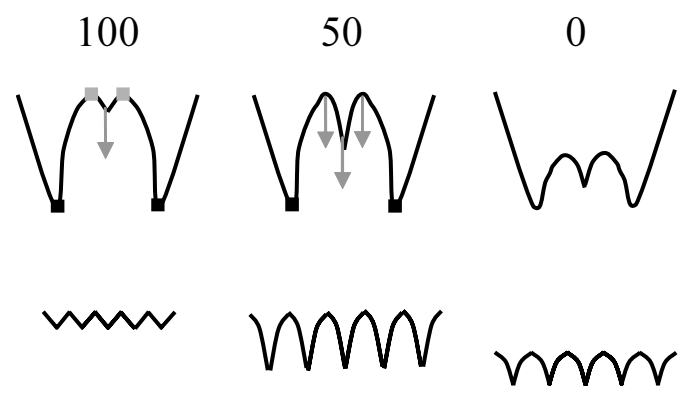

Fig. 10. Effects of genes on tube height. Top is wild type for CYC;DICH and bottom is cyc;dich. From left to right are shown reductions in DIV activity (wild type is 100, weak is 50 and null is 0). The squares at boundaries represent anchoring points (grey for ventro-lateral and black for laterodorsal). Grey arrows represent motions for generating changes in the tube height function. 
growth determinants acting non-autonomously. However, such a model would not account easily for the effects of $C Y C / D / C H$ at the dorsal boundary.

\section{Modelling growth in relation to symmetry and shape}

The descriptions above indicate that symmetry and shape are related to growth. For example, the trapezoidal shape of the lateral petal tube might come from a more rectangular and smaller structure in which a gradient of growth with a peak at the lateroventral boundary and a trough at the latero-dorsal boundary is established. Gradient of growth might be interpreted simply as differences in growth rate among petal regions. However, when differences in regional growth are considered in relation to shape, rate is insufficient to characterize growth (see, Coen et al., 2004). Two additional regional parameters are in this case required: Anisotropy which is a measure of the degree to which growth occurs preferentially in any direction and direction of growth, that is, the angle at which the principal direction of growth is oriented relative to an underlying coordinate system.

The need for these parameters and the insufficiency of growth rate can be illustrated with a hypothetical way for generating a trapeze from a rectangle (Fig. 11). The rectangle is divided in twenty identical squares each of which corresponds to a region occupying the same relative position in the trapeze (e.g., the square in line 1 , column 1 of the rectangle corresponds to the quadrangle in line 1, column 1 of the trapeze). In the case shown, the trapeze was divided in a way such that, against the interpretation above for the gradient of growth, rate increases from left to right as can be readily seen by comparing the 5 regions at the bottom. In contrast, anisotropy increases from right to left and direction changes from bottom to top and from right to left. Many other, equally arbitrary, divisions for the trapeze might have been considered (e.g., such that the final shape would be accounted for by growth rate and anisotropy varying in a manner opposite to that shown). This illustrates that differences in regional growth should incorporate rate, anisotropy and direction and that these parameters can not be deduced from the contours of the initial and final structures.

In Antirrhinum, growth parameters can be determined for each

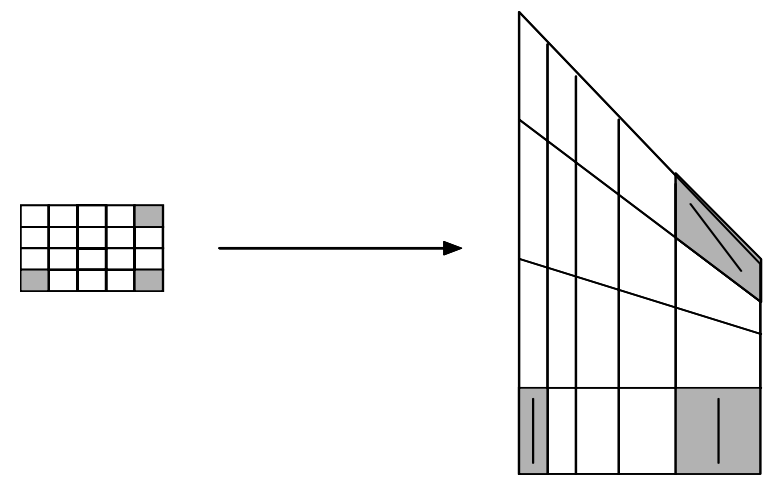

Fig. 11. Hypothetical correspondence between regions in a rectangle (e.g., schematically, the lateral petal tube early in development) and in a trapeze (mature petal). Bottom is proximal and the right side is towards dorsal. Lines inside the grey component regions indicate growth directions (e.g., relative to a vertical axis the regions at the bottom grew at $0^{\circ}$ ). of a number of regions in a structure and at successive time intervals by clonal analysis (Rolland-Lagan et al., 2003). With the values thus determined it should then be possible to construct dynamic growth models, allowing the effects of changing parameters on shape to be tested through simulation. However, for this to be feasible, a further parameter reflecting interactions between growing regions has to be considered. This is rotation, i.e., the angle by which a region turns relative to other regions per unit time. The need for this parameter can be seen by considering that if a given region grows with an unique set of values for the remaining parameters, its edges will fail to coincide with those of its neighbours after each growth step, hence causing discontinuities in the overall structure. Rotation allows for continuity to be maintained and may be particularly important in plants, where regions cannot undergo other types of motion. A dramatic case is that of leaves in cin mutants of Antirrhinum in which abnormal differences in regional growth, rather than causing discontinuities, result in undulations not seen in the normally flat leaves (Nath et al., 2003). Unlike the remaining growth parameters, rotation cannot be measured directly on the basis of clonal analysis. Instead, it is integrated in dynamic models by adjusting the values of parameters at each growth step using a spring model in which elastic properties are assigned to regions (Coen et al., 2004).

This approach has been applied to model the growth of the asymmetrical dorsal petal lobe of Antirrhinum flowers (RollandLagan et al., 2003). One conclusion was that petal lobe asymmetry is largely independent of differences in growth parameters among regions and over time. For example, simulating growth with regional parameters set at their average values results in very little change in the normal shape of the lobe. However, an increase in overall anisotropy has an effect on shape, as the petal lobe becomes stretched. Overall growth direction, in turn, influences asymmetry. This influence has however to be considered together with rotation.

Relative to an internal axis of the petal lobe, direction changes progressively during growth, resulting in asymmetry (Fig. 12A). In contrast, maintaining the initial direction throughout growth results in a nearly symmetrical lobe. Asymmetry can however be restored in a simulation in which the initial growth direction is maintained while the lobe is allowed to rotate. Rotation, rather change in direction may be what occurs when growth of the lobe is considered together with that of the tube, that is, if direction is measured relative to the proximo-distal axis of the whole flower rather than to an internal axis of the lobe (Fig. 12B). Thus, growth direction is determined globally rather than locally, suggesting that a long-range signal acts continuously to maintain growth direction (Rolland-Lagan et al., 2003).

How might growth parameters relate to the activities of genes controlling the dorsoventral pattern of Antirrhinumflowers? While this has not been investigated in detail, simulating growth of the whole dorsal petal, rather than that of only the lobe, suggested an influence of the $\mathrm{DICH}$ gene on growth rates (Coen et al., 2004). In such simulations, two types of distinctions relative to the dorsoventral axis were superimposed: One dividing the petal in dorsal and lateral halves and another dividing the petal in three regions relative to its mediolateral axis (Fig. 12C). In either case, regions differed in rates of growth with in one case greater growth in the dorsal half and in the other greater growth in the middle of the petal decreasing towards its most dorsal and most lateral 
A

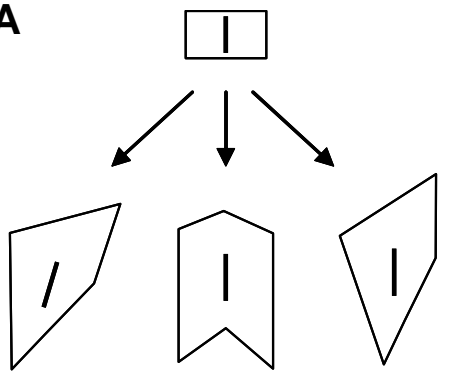

B

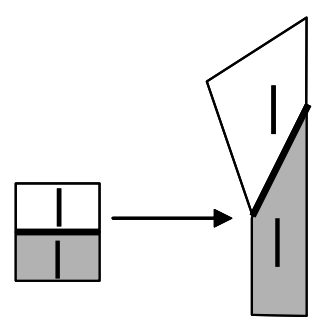

C

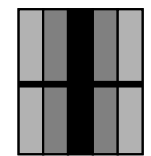

Fig. 12. Modelling dorsal petal growth. (A) Lobe growth. The wild type shape is obtained when growth direction (lines inside figures) changes on the left. At the centre a symmetrical lobe results from maintaining direction constant throughout growth. On the right direction is maintained but the lobe is allowed to rotate, restoring wild type shape. (B) Growth of lobe (white) with tube (grey). Boundary between tube and lobe (thicker line) rotates during growth while growth direction is maintained relative to the proximo-distal axis (proximal is bottom). (C) Regional distinctions in the dorsal petal: At the bottom between dorsal half (black) and lateral half (white) and at the top medio-lateral distinctions (after Coen et al., 2004).

parts. While a simulation incorporating both types of distinction resulted in a petal with the wild type shape, a simulation in which the dorsal-lateral distinction was removed produced a symmetrical dorsal petal similar to those in the backpetals or dich mutants described above.

\section{CYC IDICH in species other than Antirrhinum}

Analysing $C Y C$ counterparts in species other than Antirrhinum has provided a way of investigating evolutionary aspects of dorsoventral asymmetry of flowers. One expectation would be that similar genetic mechanisms might underlie dorsoventral asymmetry in closely related species. Thus it has been shown that the $C Y C$ gene is inactive in the peloric variant of $L$ inaria described by Linnaeus. Surprisingly, though, the cause of such inactivity is extensive methylation of the LCYC gene, that is, epimutation rather than mutation (Cubas et al., 1999b). Studies with other species with bilaterally symmetrical flowers, again suggest the involvement of $C Y C$-like genes in determining dorsoventral asymmetry. In addition, differences in patterns of expression of CYC/D/CH counterparts may account for variation in morphological details of flowers. An example comes from Mohavea confertiflora, a species with bilaterally symmetrical flowers belonging to the same family as Antirrhinum (Scrophulariaceae). The two species have the same number of organ primordia occupying the same relative positions. One difference, however, is that development of both the dorsal and lateral stamen primordia is arrested in Mohavea, whereas only the dorsal primordium is arrested in Antirrhinum. This correlates with expansion of the domain of $C Y C / D / C H$ expression towards lateral positions in Mohavea, relative to what is observed in Antirrhinum (Hileman, et al., 2003).

What, however, might be the role of $C Y C$-like genes in species that are distantly related to and differ widely from Antirrhinum in morphology? This question has been addressed for maize and Arabidopsis. In the case of maize, it has been found that a TCP gene, TEOSINTE BRANCHED1 (TB1), controls plant architecture (Doebley et al., 1997). Maize and its ancestor Teosinte differ in branching pattern, with maize producing lateral female inflorescences where branches grow in Teosinte. This major difference is attributable to variation in the expression of the $T B 1$ gene, correlating with suppression of axillary branch growth (Hubbard et al., 2002). In Antirrhinum, $C Y C$ also has a part in suppressing growth. Thus, $C Y C$ and $T B 1$ appear to have a conserved role in regional growth regulation. In addition, genes activities relate in both cases to apical meristem behaviour, although $C Y C$ has no apparent effect on branching.

A perhaps surprising finding was that the Arabidopsis CYC counterpart is expressed in a dorsal domain of the floral meristem, similarly to what is observed in Antirrhinum, even though the Arabidopsis meristem is destined to produce radially symmetrical flowers (Cubas et al., 2001). This provides an important clue to understanding how dorsoventral asymmetry of flowers may have evolved repeatedly. Presumably, an ancient asymmetry in $C Y C$ expression was already present in radially symmetrical flowers of a common ancestor of Antirrhinum and Arabidopsis. Elaboration of this incipient asymmetry, through changes in genetic interactions, would have then resulted in morphological manifestation of dorsoventral asymmetry independently in various lineages. It remains unclear, however, what ancient role CYC might have played.

As noted before, Antirrhinumand Arabidopsisproduce flowers in lateral positions with organs aligned relative to the dorsoventral axis (which is an axis of asymmetry in Antirrhinum and not in Arabidopsis). Thus, in both cases the dorsal domain expressing $C Y C$ lies nearer to the apical inflorescence meristem than the rest of the flower. As the apex itself shows centre to periphery asymmetry, establishment of dorsal-specific expression might reflect that $C Y C$ interprets signals related to apex asymmetry, rather than to floral identity. This has been addressed in Antirrhinum by examining CYC expression in the meristem-identity mutant floricaula (flo). Unlike in wild type, in which the inflorescence meristem produces bracts in its periphery, which subsequently will subtend floral meristems, bracts of flo mutant plants subtend further inflorescence meristems (Coen et al., 1989). These secondary inflorescence meristems do themselves produce bracts that will in turn subtend tertiary inflorescence meristems. Despite their inflorescence identities, these secondary and tertiary meristems express $C Y C$ in domains that are always located dorsally relative to the meristems from which they originated (Clark and Coen, 2002). Thus, dorsal-specific expression of $C Y C$ can be established independently of floral identity. Dorsal-specific expression of the Arabidospsis CYC gene might similarly reflect an apical influence. Unlike in Antirrhinum, how- 
ever, where CYC expression persists through petal and stamen development, the Arabidospis CYC gene is expressed only transiently in the flower meristem, perhaps accounting for the lack of dorsoventral asymmetry of Arabidopsis flowers (Cubas et al., 2001). This transient expression might however be sufficient for suppressing growth or for bringing organ primordia into register with the dorsoventral axis of the flower, similarly to the parts played by $C Y C$ early in Antirrhinum flower development.

\section{Final remarks}

The studies above indicate that it is possible to explain shape and symmetry by linking gene activities to growth, considered in a quantitative framework. Much, however, remains to be understood on how genes such as $C Y C, D / C H$ and $D I V$ more precisely relate to growth parameters and on how they interact with other genes controlling growth. Amongst these are, for example, determinants of mediolateral distinctions in floral organs, as proposed in the model accounting for the effect of $\mathrm{DICH}$ (Coen et al., 2004). In radially symmetrical mutants only this type of distinction remains in all organs giving rise to a periodic function, the amplitude of which appears to depend on DIV (Fig. 11). However, what the determinants of such function might be is unknown.

Small quantitative change in growth parameters, leading to subtle modifications of shapes, may have provided finely grained variation on which large morphological differences have built during evolution. The origin of that variation might be studied using diverse flower shape mutants that have been identified in Antirrhinum through over a century of genetic research (Stubbe, 1966, Schwarz-Sommer etal., 2003). For many of these mutants, however, differences relative to wild type remain poorly described. This reflects that some of those differences are subtle and, perhaps mostly, that so far there has not been an adequate common framework for characterizing shape. Progress in modelling growth and in quantitative descriptions of shape might provide that needed framework.

\section{Acknowledgements}

We thank Enrico Coen for reading the manuscript. Work of our group was funded by FCT (Sapiens 36221)

\section{References}

ALMEIDA, J., ROCHETA, M. and GALEGO, L. (1997). Genetic control of flower shape in Antirrhinum majus. Development 124: 1387-1392.

BATESON, W. (1894). Materials for the study of variation treated with especial regard to discontinuity in the origin of species. MacMillan, New York and London.

BAUR, E. (1910). Vererbungs- und Bastardierungsversuche mit Antirrhinum. Z. f. indukt. Abst.-u. Vererbungs/. 3: 34-98.

BRADLEY, D., CARPENTER, R., SOMMER, H., HARTLEY, N. and COEN, E. (1993). Complementary floral homeotic phenotypes result from opposite orientations of a transposon at the plena locus of Antirrhinum. Cell 72: 85-95.

BRADLEY, D., CARPENTER, R., COPSEY, L., VINCENT, C., ROTHSTEIN, S. and COEN, E. (1996). Control of inflorescence architecture in Antirrhinum. Nature 379: 791-797.

CARPENTER, R. and COEN, E. (1990). Floral homeotic mutations produced by transposon-mutagenesis in Antirrhinum majus. Genes Dev. 4: 1483-1493.

CARPENTER, R., COPSEY, L., VINCENT, C., DOYLE, S., MAGRATH, R. and COEN, E. (1995). Control of flower development and phyllotaxy by meristem identity genes in Antirrhinum. Plant Cell 7: 2001-2011.
CLARK, J. and COEN, E. (2002). The cycloidea gene can respond to a common dorsoventral prepattern in Antirrhinum. Plant J. 30 (6): 639-648.

COEN, E. (1991). The role of homeotic genes in flower development and evolution. Annu. Rev. Plant Physiol. Plant Mol. Biol. 42: 241-279.

COEN, E. (1996). Floral symmetry. EMBO J. 15: 6777-6788.

COEN, E. and MEYEROWITZ, E. (1991). The war of the whorls: Genetic interactions controlling flower development. Nature 353: 31-37.

COEN, E. and NUGENT, J.M. (1994). Evolution of floral symmetry. Development Supplement: 107-116.

COEN, E., NUGENT, J., LUO, D., BRADleY, D., CUBAS, P., CHADWICK, M., COPSEY, L. and CARPENTER, R. (1995). Evolution of floral symmetry. Philos. Trans. R. Soc. Lond. B Biol. Sci. 350: 35-38.

COEN, E., ROLLAND-LAGAND, A-G., MATHEWS, M., BANGHAM, A. and PRUSINKIEWICZ, P. (2004). The genetics of geometry. Proc. Natl. Acad. Sci. USA. 101: 4728-4735

CRONK, Q.C.B. (2001). Plant evolution and development in a post-genomic context. Nat. Rev. Genet. 2 (8): 607-619.

CUBAS, P., LAUTER, N., DOEBLEY, J. and COEN, E. (1999a). The TCP domain: a motif found in proteins regulating plant growth and development. Plant J. 18: 215-222.

CUBAS, P., VINCENT, C. and COEN, E. (1999b). An epigenetic mutation responsible for natural variation in floral symmetry. Nature 401: 157-161.

CUBAS, P., COEN, E. and ZAPATER, J. (2001). Ancient asymmetries in the evolution of flowers. Curr. Biol. 11: 1050-1052.

DARWIN, C. (1868). The variation of animals and plants under domestication, vol. 2. Murray, London.

DE VRIES, H. (1901). Die Mutationstheorie. 1. Verlag von Veit u. Co., Leipzig.

DOBLEY, J., STEC, A. and HUBBARD, L. (1997). The evolution of apical dominance in maize. Nature 386: 485-488.

ENDRESS, P.K. (2001). Evolution of floral symmetry. Curr. Opin. Plant Biol. 4: 8691.

FARMER, D.W. (1996). Groups and Symmetries - A Guide to Discovering Mathematics. The Americam Mathematical Society.

GALEGO, L. and ALMEIDA, J. (2002). Role of DIVARICATA in the control of dorsoventral asymmetry in Antirrhinum flowers. Genes Dev. 16: 880-891.

GUSTAFSSON, A. (1979). Linnaeus' peloria - history of a monster. Theor. Appl. Genet. 54: 241-248.

HILEMAN, L.C., KRAMER, E.M. and BAUM, D:A. (2003). Differential regulation of symmetry genes and the evolution of floral morphologies. Proc. Natl. Acad. Sci USA. 100: 12814-12819.

HUBBARD, L., MCSTEEN, P., DOBLEY, J. and HAKE, S. (2002). Expression patterns and mutant phenotype of teosinte branched1 correlate with growth suppression in maize and teosinte. Genetics 162:1927-1935.

HUDSON, A. (2000). Development of symmetry in plants. Annu Rev Plant Physiol Plant Mol Biol. 51: 349-370.

LINNAEUS, C. (1749). De Peloria. Amoenitates Academy, Uppsala, Sweden.

LUO, D., CARPENTER, R., VINCENT, C., COPSEY, L. and COEN, E. (1996). Origin of floral asymmetry in Antirrhinum. Nature 383: 794-799.

LUO, D., CARPENTER, R., COPSEY, L., VINCENT, C., CLARK, J. and COEN, E. (1999). Control of organ asymmetry in flowers of Antirrhinum. Cel/ 99:367-376.

NATH, U., CRAWFORD, B., CARPENTER, R. and COEN, E. (2003). Genetic control of surface curvature. Science 299: 1404-1407.

NEAL, P., DAFNI, A. and GIURFA, M. (1998). Floral symmetry and its role in plantpollinator systems: terminology, distribution and hypotheses. Annu. Rev. Ecol. System. 29: 345-373.

ROLLAND-LAGAN, A-G., BANGHAM, J.A. and COEN, E. (2003). Growth dynamics underlying petal shape and asymmetry. Nature 422: 161-163.

RUDALL, P. And BATEMAN, R. (2004). Evolution of zygomorphy in monocot flowers: iterative patterns and developmental constraints. New Phytol. 162: 2544.

SCHEINERMAN, E.R. (2000). Mathematics. A Discrete Introduction. Brooks/Cole Publishing Company, USA.

SCHICK, R. and STUBBE, H. (1934). Die gene von Antirrhinum majus. III. (zugleich 
ein beitrag zur genetischen nomenklatur). Z. f. induct. Abst. - v. Vererbungs/. 66: 425-462.

SCHWARZ-SOMMER, Z., HUIJSER, P., NACKEN, W., SAEDLER, H. and SOMMER, H. (1990). Genetic control of flower development by homeotic genes in Antirrhinum majus. Science 250: 931-936.

SCHWARZ-SOMMER, Z., DAVIES, B. and HUDSON, A. (2003). An everlasting pioneer: the story of Antirrhinum genetics. Nat. Rev. Genet. 4 (8): 657-666.

SESSIONS, A. and YANOFSKY, M. (1999). Dorsoventral patterning in plants. Genes Dev. 9: 1051-1054.

SOMMER, H., BELTRAN, J-P., HUIJSER, P., PAPE, H., LONNIG, W-E., SAEDLER, H. and SCHWARZ-SOMMER, Z. (1990). Deficiens, a homeotic gene involved in the control of flower morphogenesis in Antirrhinum majus : the protein shows homology to transcription factors. EMBO J. 9: 605-613.
STUBBE, H. (1966). Genetik und Zytologie von Antirrhinum L. sect Antirrhinum. Veb Gustav Fischer Verlag, Jena.

VINCENT, C., CARPENTER, R. and COEN, E. (1995). Cell lineage patterns and homeotic gene activity during Antirhinum flower development. Curr. Biol. 5:1449-1458.

VINCENT, C.A. and COEN, E.S. (2004). A temporal and morphological framework for flower development in Antirrhinum majus. Can. J. Bot. 82 (5): 681-690.

WAITES, R., SELVADURAI, H., OLIVER, I. and HUDSON, A. (1998). The PHANTASTICA gene encodes a MYB transcription factor involved in growth and dorsoventrality of lateral organs in Antirrhinum. Cell 93: 779-789.

WAITES, R. and HUDSON, A. (2001). The Handlebars gene is required with Phantastica for dorsoventral asymmetry of organs and for stem cell activity in Antirrhinum. Development 128: 1923-1931. 